and outcome. All patients had symptoms of synucleinopathy, manifesting with autonomic failure, REM behavior disorder, and parkinsonism. Four met criteria for idiopathic PD, and one was diagnosed with pure autonomic failure but had concomitant symptoms of parkinsonism and REM sleep behavior disorder. RESULTS/ANTICIPATED RESULTS: Our patients had no significant cognitive or behavioral symptoms before the initiation of droxidopa. The average decrease in blood pressure upon standing was $27 \mathrm{mmHg}$ systolic and $17 \mathrm{mmHg}$ diastolic. Behavioral disturbances were observed early in the titration period and at relatively low doses of droxidopa (total daily doses ranging from 300 to $800 \mathrm{mg} /$ day; droxidopa therapeutic dose range $900-1800 \mathrm{mg} / \mathrm{d}$ ). The most common symptoms reported were mania, irritability, and confusion. Symptoms resolved with dose reduction in 4 patients, and droxidopa was discontinued in I patient due to persistent irritability. No other medical comorbidities or alternative etiologies were identified to explain these effects. DISCUSSION/ SIGNIFICANCE OF IMPACT: Droxidopa is a prodrug designed to act peripherally, but may also have important, yet poorly described, central effects. We hypothesize that these behavioral manifestations result from an "overdose" of key NE networks linking orbitofrontal and mesolimbic regions. Further studies are warranted to better characterize central NE effects in patients treated with droxidopa.

\section{Delirium and catatonia: Age matters}

\section{Jo E. Wilson', Richard Carlson', Maria C. Duggan ${ }^{2,3}$, Pratik} Pandharipande ${ }^{4}$, Timothy D. Girard ${ }^{5}$, Li Wang ${ }^{6}$, Jennifer L. Thompson ${ }^{6}$, Rameela Chandrasekhar ${ }^{6}$, Andrew Francis ${ }^{7}$, Stephen E. Nicolson ${ }^{8}$, Robert S. Dittus ${ }^{3,9}$, Stephan Heckers' and E. W. Ely ${ }^{3,9}$ For the Delirium and Catatonia (DeCat) Prospective Cohort Investigation ' Department of Psychiatry, Vanderbilt University Medical Center, Nashville, TN, USA; ${ }^{2}$ Department of Medicine, Division of General Internal Medicine and Public Health, Vanderbilt University School of Medicine, Nashville, TN, USA; ${ }^{3}$ Veteran's Affairs TN Valley, Geriatrics Research, Education and Clinical Center (GRECC); ${ }^{4}$ Department of Anesthesiology and Critical Care; ${ }^{5}$ Department of Critical Care Medicine, Clinical Research, Investigation, and Systems Modeling of Acute illness (CRISMA) Center, University of Pittsburgh School of Medicine, Pittsburgh, PA, USA; ${ }^{6}$ Department of Biostatistics, Vanderbilt University Medical Center, Nashville, TN, USA; ${ }^{7}$ Department of Psychiatry, Penn State Medical School, Hershey Medical Center, Hershey, PA, USA; ${ }^{8}$ Department of Psychiatry, Beth Israel Deaconess Hospital-Plymouth, Plymouth, MA, USA; ${ }^{9}$ Department of Medicine, The Center for Health Services Research, Division of Pulmonary and Critical Care, Vanderbilt University Medical Center, Nashville, TN, USA

OBJECTIVES/SPECIFIC AIMS: Background: Delirium is a well described form of acute brain organ dysfunction characterized by decreased or increased movement, changes in attention and concentration as well as perceptual disturbances (i.e., hallucinations) and delusions. Catatonia, a neuropsychiatric syndrome traditionally described in patients with severe psychiatric illness, can present as phenotypically similar to delirium and is characterized by increased, decreased and/or abnormal movements, staring, rigidity, and mutism. Delirium and catatonia can co-occur in the setting of medical illness, but no studies have explored this relationship by age. Our objective was to assess whether advancing age and the presence of catatonia are associated with delirium. METHODS/STUDY POPULATION: Methods: We prospectively enrolled critically ill patients at a single institution who were on a ventilator or in shock and evaluated them daily for delirium using the Confusion Assessment for the ICU and for catatonia using the Bush Francis Catatonia Rating Scale. Measures of association (OR) were assessed with a simple logistic regression model with catatonia as the independent variable and delirium as the dependent variable. Effect measure modification by age was assessed using a Likelihood ratio test. RESULTS/ANTICIPATED RESULTS: Results: We enrolled 136 medical and surgical critically ill patients with 452 matched (concomitant) delirium and catatonia assessments. Median age was 59 years (IQR: $52-68)$. In our cohort of I 36 patients, 58 patients $(43 \%)$ had delirium only, $4(3 \%)$ had catatonia only, 42 (3I\%) had both delirium and catatonia, and $32(24 \%)$ had neither. Age was significantly associated with prevalent delirium (i.e., increasing age associated with decreased risk for delirium) $(p=0.04)$ after adjusting for catatonia severity. Catatonia was significantly associated with prevalent delirium $(p<0.000 \mathrm{I})$ after adjusting for age. Peak delirium risk was for patients aged 55 years with 3 or more catatonic signs, who had 53.4 times the odds of delirium $(95 \% \mathrm{Cl}: 16.06,176.75)$ than those with no catatonic signs. Patients 70 years and older with 3 or more catatonia features had half this risk. DISCUSSION/SIGNIFICANCE OF IMPACT: Conclusions: Catatonia is significantly associated with prevalent delirium even after controlling for age. These data support an inverted U-shape risk of delirium after adjusting for catatonia. This relationship and its clinical ramifications need to be examined in a larger sample, including patients with dementia. Additionally, we need to assess which acute brain syndrome (delirium or catatonia) develops first.

Depression, anxiety, and planning for the future: Associations with advance care planning Ryan McMahan', Evan Walker ${ }^{2}$ and Rebecca Sudore ${ }^{3}$

' San Francisco School of Medicine, University of California, CA, USA;

${ }^{2}$ Department of Internal Medicine, University of California, San Francisco, CA, USA; ${ }^{3}$ Department of Medicine, San Francisco VA Medical Center, Division of Geriatrics, University of California, San Francisco, CA, USA

OBJECTIVES/SPECIFIC AIMS: Millions of diverse, older adults live with serious and chronic illness for which they will face complex, ongoing medical decisions. Advance care planning (ACP) has been conceptualized as a health behavior that supports adults in understanding and sharing their values, goals, and preferences for future medical care. Depression and anxiety are known barriers to participation in health behaviors. It is unknown whether depression and anxiety are associated with ACP participation or with patients' values for future medical care. Understanding whether depression and anxiety are associated with ACP would be important to tailor ACP interventions. METHODS/STUDY POPULATION: In total, 908 English-speaking and Spanish-speaking participants $\geq 55$ years of age were recruited from a San Francisco county hospital. We measured depression (Patient Health Questionnaire 8-item scale) and anxiety (Generalized Anxiety Disorder 7-item scale), dichotomized into none-to-mild Versus moderate-to-severe. We measured ACP engagement using a validated survey of Behavior Change Processes (e.g., knowledge, self-efficacy, readiness; 5-point Likert) and Action Measures (e.g., ask, discuss, and document one's wishes; yes/ no). We elicited values concerning life extension categorized as "life is always worth living no matter the health situation" Versus "some health situations would make life not worth living." To explore associations, we used $\chi^{2}$, Mann-Whitney tests, linear and logistic regressions. RESULTS/ANTICIPATED RESULTS: Mean participant age was 64 years $\pm 6,80 \%$ were non-White, $40 \%$ had limited literacy, $45 \%$ were Spanish-speaking, and the prevalence of depression and anxiety was $12 \%$ and $10 \%$, respectively. Depression and anxiety were not associated with ACP Engagement, $p>0.05$. However, participants with depression had an increased odds of reporting "some health situations would make life not worth living" than those not depressed, $p=0.02$. In multivariate linear and logistic regression, controlling for age, gender, literacy, and health status, having depression increased the odds of not valuing life extension OR 2.9 (Cl: I.7-4.9) Anxiety was not associated with values concerning life extension, $p>0.05$. DISCUSSION/SIGNIFICANCE OF IMPACT: Depression and anxiety were not associated with prior ACP engagement suggesting engaging patients in ACP does not increase these conditions. However, depression was associated with an increased odds of not valuing life extension and, therefore, may influence treatment choices. Longitudinal randomized controlled trials of an ACP intervention are currently underway to investigate these associations further.

2166

Development of a statin risk communication tool for use in cancer survivors: A pilot

Nirupa J. Raghunathan, Nassim Anderson, Emily Tonorezos and

Deborah Korenstein

Memorial Sloan Kettering Cancer Center

OBJECTIVES/SPECIFIC AIMS: There are currently over a million survivors of childhood, adolescent, and young adult cancer in the United States, many of whom were treated with radiation therapy. Chest radiation with fields including the coronary arteries is a risk factor for cardiovascular disease. Of note, survivors are often unaware of this increased cardiovascular disease risk or, if they are aware, do not know how to mitigate the risk. Visual aids and communicating risk in terms of absolute risk reductions are shown to improve patients' understanding. The Institute of Medicine recommends use of decision aids to optimize patient discussions of benefits and harms of therapies. Our goal is to develop and pilot test a statin therapy risk communication tool for use in high-risk cancer survivors to improve shared decision making and patient knowledge of coronary artery disease risk. METHODS/ STUDY POPULATION: Participants were recruited from the adult long-term follow-up clinic at Sloan Kettering Cancer Center into 2 arms, usual care Versus 